\title{
G

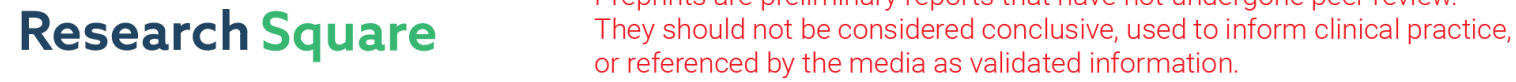 \\ miRNA Profiling in Human Precision-cut Lung Slices (PCLS))
}

Monika Niehof ( $\square$ monika.niehof@item.fraunhofer.de )

Fraunhofer ITEM https://orcid.org/0000-0001-5687-3052

\section{Stella Reamon-Buettner}

Fraunhofer Institute for Toxicology and Experimental Medicine: Fraunhofer-Institut fur Toxikologie und Experimentelle Medizin ITEM

\section{Olga Danow}

Fraunhofer Institute for Toxicology and Experimental Medicine ITEM: Fraunhofer-Institut fur Toxikologie und Experimentelle Medizin ITEM

\section{Tanja Hansen}

Fraunhofer Institute for Toxicology and Experimental Medicine ITEM: Fraunhofer-Institut fur Toxikologie und Experimentelle Medizin ITEM

\section{Katherina Sewald}

Fraunhofer Institute for Toxicology and Experimental Medicine ITEM: Fraunhofer-Institut fur Toxikologie und Experimentelle Medizin ITEM

\section{Research note}

Keywords: miRNA extraction, RTqPCR, microarray, miRNA array, RNA quality, lung tissue, lung material, ex vivo, PCLS, organotypic tissue

Posted Date: March 31st, 2021

DOI: https://doi.org/10.21203/rs.3.rs-362859/v1

License: (c) (i) This work is licensed under a Creative Commons Attribution 4.0 International License. Read Full License 


\section{Abstract}

Objective

Human precision cut lung slices (PCLS) are widely used as an ex vivo model system for drug discovery and development of new therapies. PCLS reflect the functional heterogeneity of lung tissue and possess relevant lung cell types. We thus determined the use of PCLS in studying non-coding RNAs notably miRNAs, which are important gene regulatory molecules. Since miRNAs play key role as mediators of respiratory diseases, they can serve as valuable prognostic or diagnostic biomarkers, and in therapeutic interventions, of lung diseases. A technical limitation though is the vast amount of agarose in PCLS which impedes (mi)RNA extraction by standard procedures. Here we modified our recently published protocol for RNA 29 isolation from PCLS to enable miRNA readouts.

Results

The modified method relies on the separation of lysis and precipitation steps, and a clean-up procedure with specific magnetic beads. We obtained successfully quality miRNA amenable for downstream applications such as RTqPCR and whole transcriptome miRNA analysis.

Comparison of miRNA profiles in PCLS with published data from human lung, identified all important miRNAs regulated in IPF, COPD, asthma or lung cancer. Therefore, this shows suitability of the method for analyzing miRNA targets and biomarkers in the valuable human 38 PCLS model.

\section{Full-text}

Due to technical limitations, full-text HTML conversion of this manuscript could not be completed. However, the manuscript can be downloaded and accessed as a PDF.

\section{Figures}


a
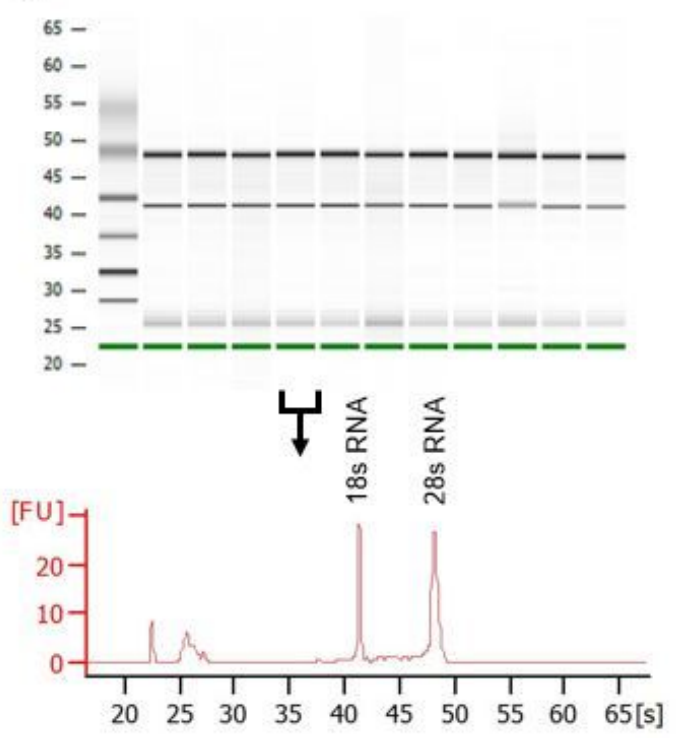
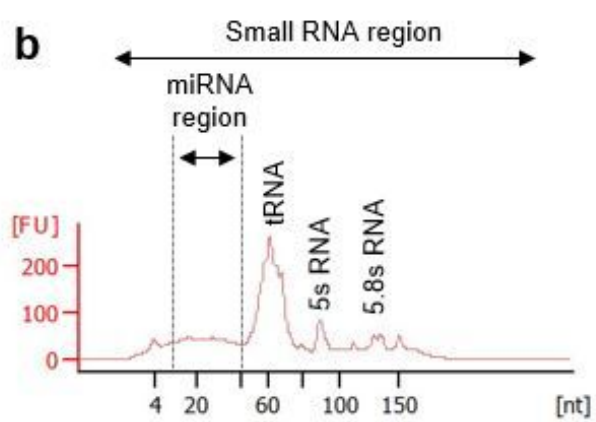

C

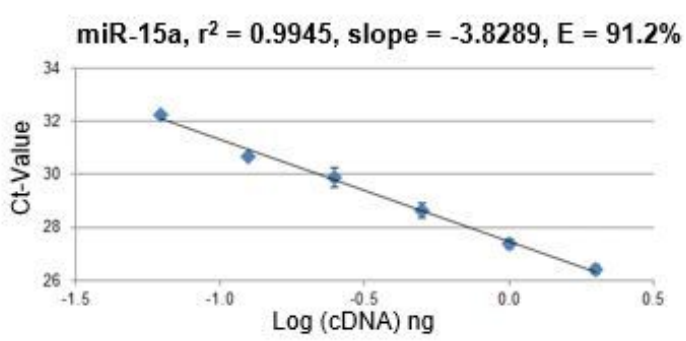

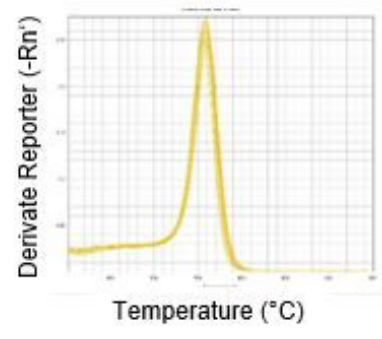

\section{Figure 1}

Assessment of RNA quality for human PCLS. (a) Representative bioanalyzer results using Agilent RNA 600 Nano assay showing the integrity of RNA isolated from 10 different human PCLS samples (virtual RNA gel format and electropherogram depicting fluorescence units versus run time in seconds). (b) Representative bioanalyzer results using Agilent Small RNA assay visualizing the presence of small RNAs (electropherogram depicting fluorescence units versus run time in seconds). (c) miScript PCR assay for miR-15a, calibration curve and melting curve using several human PCLS cDNA dilutions. 
a

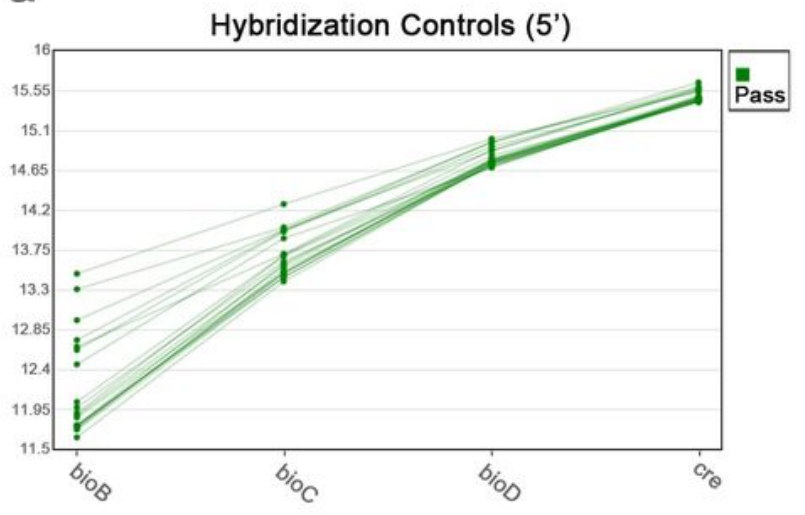

C

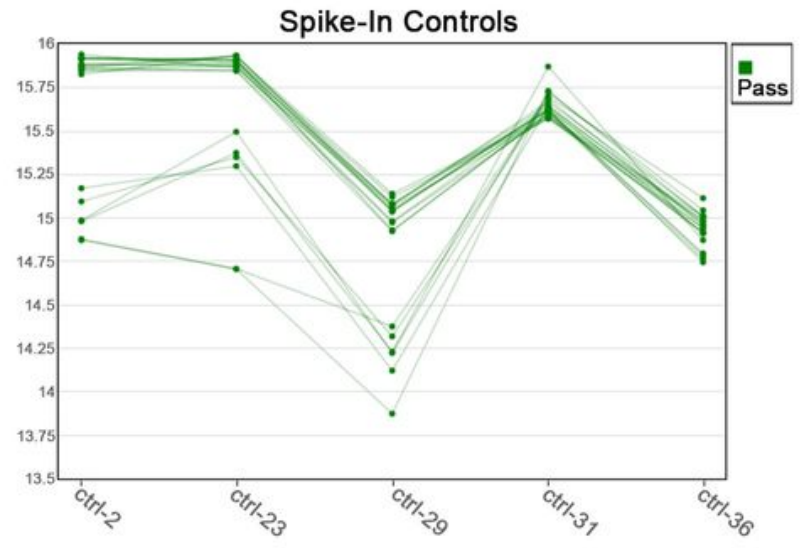

f

e

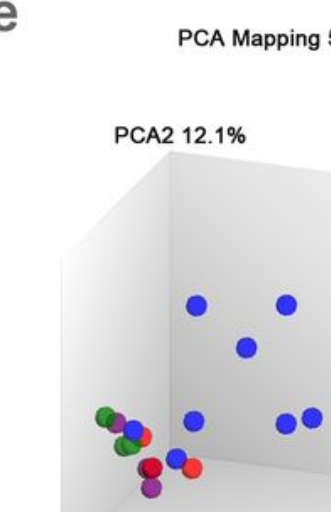

PCA3 7.5\% b

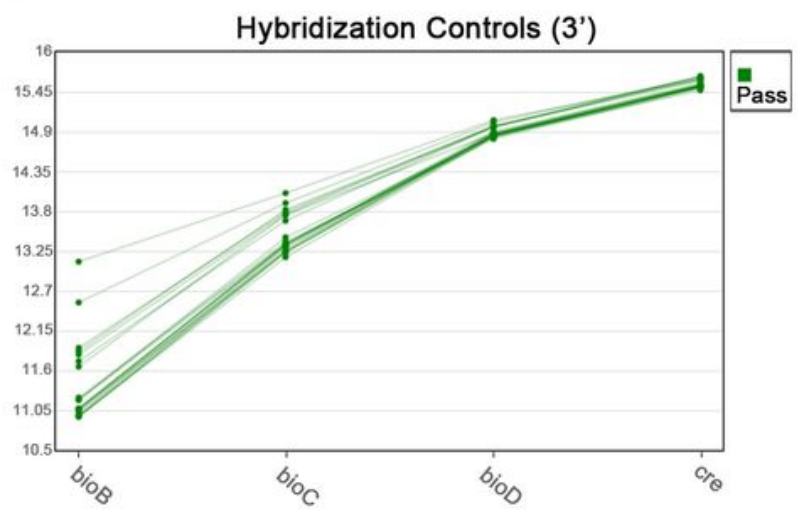

d

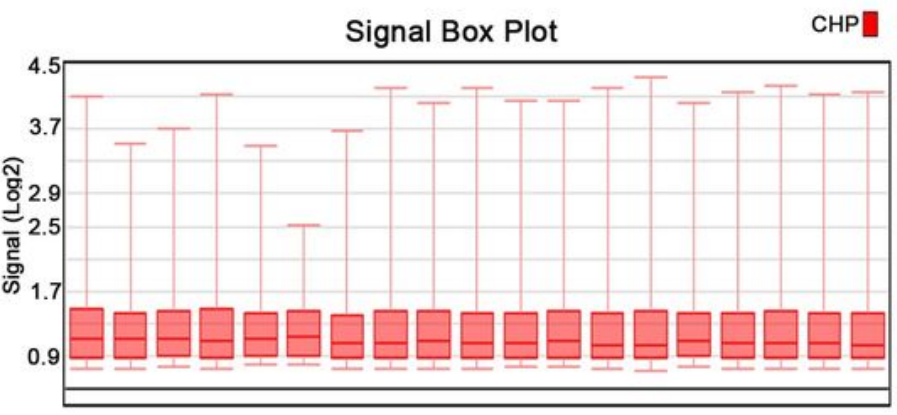

Hierarchical Clustering

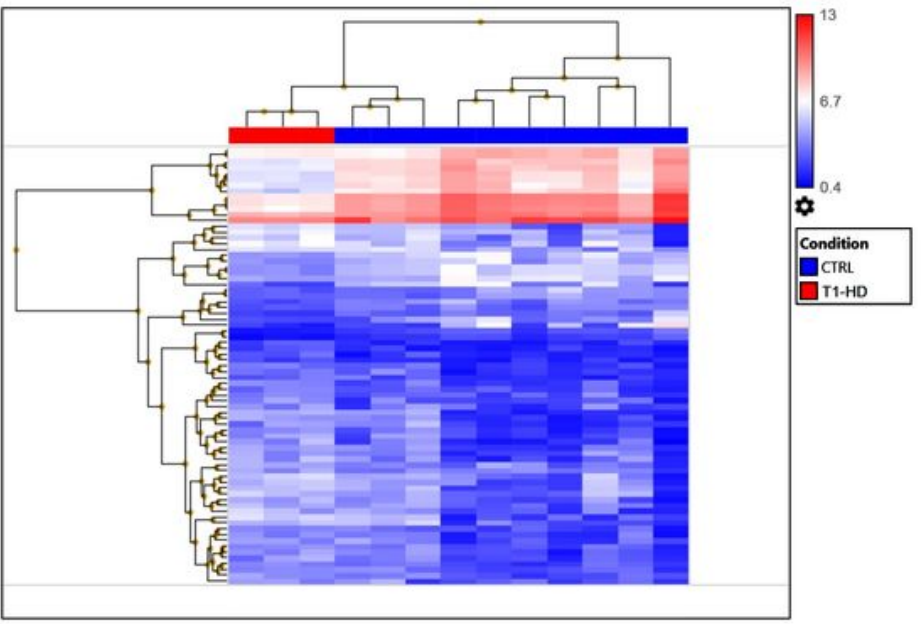

\section{Figure 2}

Quality control of miRNA data. Affymetrix microarrays were subjected to quality control criteria specified in Transcriptome Analysis Console (TAC 4.0), i.e. (a, b) 5' and 3' hybridization controls, (c) spike-in controls and (d) signal box plot. (e) Principal component analysis (PCA) mapping of the miRNA data, showing majority of untreated control samples in one group and treated samples in another group. (f) 
Hierarchical clustering of filtered genes (Fold Change $<-2$ or $>2$, P-Value $<0.05$ ) in samples treated with T1-HD vs. untreated control.

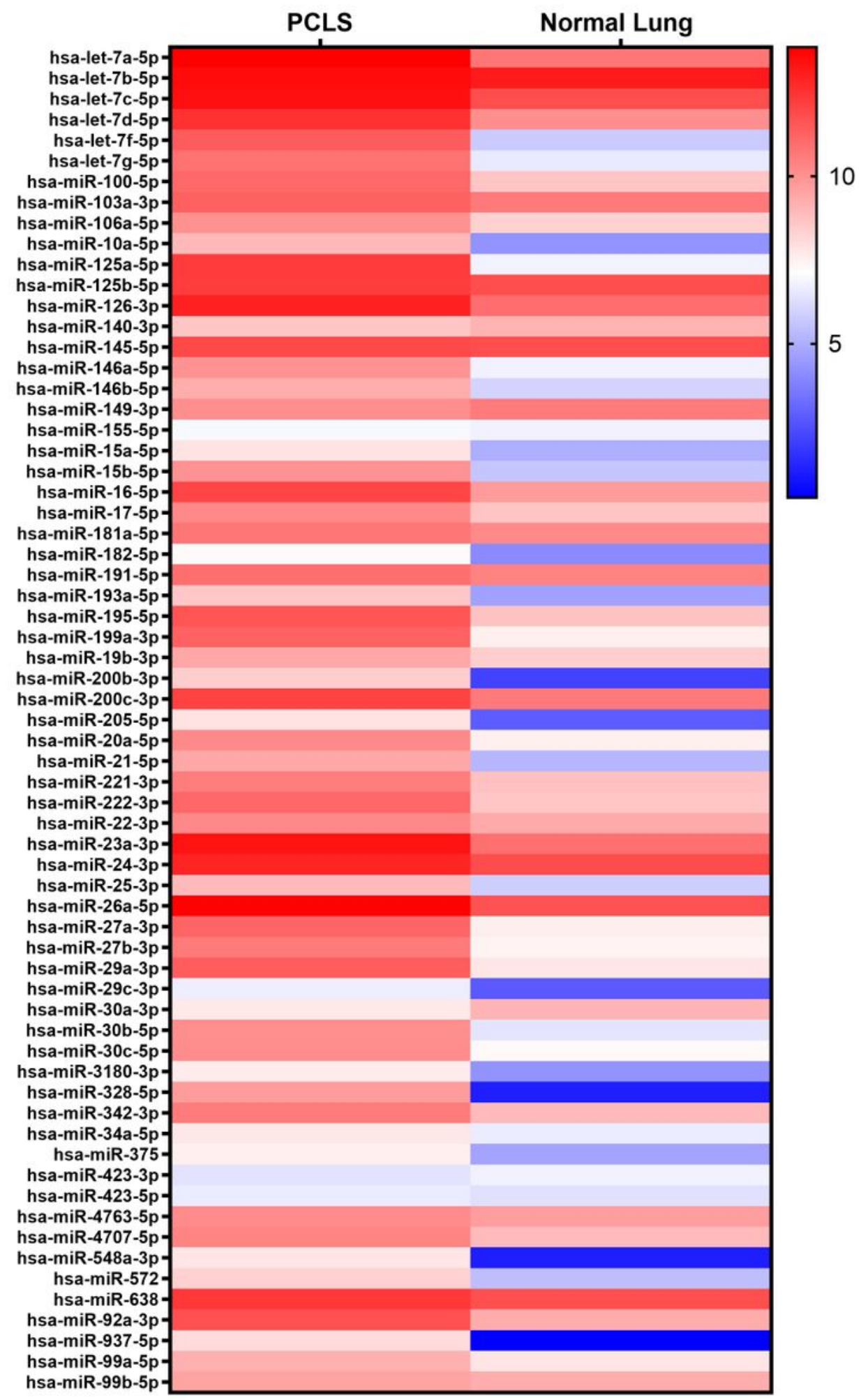

Figure 3

Examples of lung-disease related miRNAs obtained in human PCLS and their level of expression as compared to normal lung samples (GSE81293). Heatmap was based on average signal intensities (log2 values) after microarray analysis and generated using GraphPad Prism 8.3.1. 


\section{Supplementary Files}

This is a list of supplementary files associated with this preprint. Click to download.

- NiehofAdditionalfile1.docx

- NiehofAdditionalfile2.docx

- NiehofAdditionalfile3.docx

- NiehofAdditionalfile4.docx 